\title{
3-Level DWT Image Watermarking Against Frequency and Geometrical Attacks
}

\author{
Asma Ahmad \\ Department of Electronics \& Telecommunication Engineering, Shri Shankaracharya Technical Campus Bhilai, India \\ Email:asma.ahmad28@gmail.com
}

G.R.Sinha

Professor and Associate Director, Faculty of Engineering \& Tech, Shri Shankaracharya Technical Campus Bhilai, India Email: drgrsinha@ieee.org, ganeshsinha2003@gmail.com

\author{
Nikita Kashyap \\ Assistant Professor, Department of Electronics \& Telecomm. Engg, Lakhmichand Institute of Technology Bilaspur, India \\ Email: nikita2010k@gmail.com
}

\begin{abstract}
This paper introduces a robust image watermarking technique for the copyright protection. The proposed method is based on 3-level discrete wavelet transform (DWT). Encoded secret image using spiral scanning is hidden by alpha blending technique in LL sub bands. During embedding process, secret image is dispersed within LL band depending upon alpha value. Encoded secret images are extracted and decoded to recover the original secret image. The experimental results demonstrate that the watermarks generated with the proposed algorithm are invisible and the quality of watermarked image and the recovered image are improved. The scheme is found robust to various image processing attacks such as JPEG compression, Gaussian noise, blurring, median filtering and rotation.
\end{abstract}

Index Terms-Image watermarking, 3-level DWT, wavelet transform, attacks, mean square error (MSE), peak signal to noise ratio (PSNR).

\section{INTRODUCTION}

The development of effective digital image copyright protection methods have recently become an urgent and necessary requirement in the multimedia industry due to the ever-increasing unauthorized manipulation and reproduction of original digital objects. The new technology of digital watermarking has been advocated by many researchers as the best method to such multimedia copyright protection problem [1]. It is expected that digital watermarking would have a widerange of practical applications in digital cameras, medical imaging, image databases, and video-on-demand systems etc.

Digital watermarking deals with information hiding which is used to hide proprietary information in digital media like photographs, digital music, or digital video [2$3]$. The ease with which digital content can be exchanged over the Internet has created copyright infringement issues. Copyrighted material can be easily exchanged over peer-to-peer networks, and it has caused major concerns to content providers engaged in producing the contents.

For an efficient watermarking method, it should be robust to compression, filtering, rotation, scaling cropping, and collusion attacks among many other digital processing operations. The existing digital image watermarking techniques can be grouped into two major classes namely Spatial domain Watermarking and Transform Domain Watermarking techniques. In comparison with spatial domain techniques [4], transform-domain watermarking techniques (DWT) are generally more effective in terms of the imperceptibility and robustness requirements of digital watermarking algorithms [5-6]. A transform domain technique is proposed which shows greater robustness to common signal distortions. The main advantage of the proposed wavelet-based technique lies in the method used to embed the watermark in low frequency band using blending technique. Performance improvements in DWTbased digital image watermarking algorithms could be obtained by increasing the level of DWT.

\section{RELATED WORK}

This section highlights research works related to digital watermarking used for digital images. The existing contributions in terms of their findings and limitations have been reported.

Mistry [6] introduced digital watermarking methodsSpatial domain (like LSB) and transform domain (like DCT, DWT) methods. The spatial domain is the normal image space, in which a change in position in image directly projects to a change in position in space. Ex.Least Significant bit (LSB) method. Transform Domain method produce high quality watermarked image by first transforming the original image into the frequency domain by the use of Fourier Transform, Discrete Cosine Transform (DCT) or Discrete Wavelet transforms (DWT). It was observed that that transform watermarking is 
comparatively much better than the spatial domain encoding. Transform based methods are very efficient and having more robustness for image processing attacks.

Gunjal [7] introduced a complete overview of digital image watermarking in spatial as well as transform domain. This paper focuses on quality factors essential for good quality watermarking, performance evaluation metrics (PSNR and Correlation Factors) and possible attacks. Overview of several methods with spatial and Transform domain watermarking was presented along with detailed mathematical background and their implementations. Comparative results of Digital Image Watermarking using least significant bit (LSB) method, discrete cosine transform (DCT) and discrete wavelet transform (DWT) method report that DWT based technique could achieve more robustness than other techniques. Hence more number of research works are being carried out in this category of watermarking technique.

Blossom et al. [8] proposed a DCT based watermarking scheme which provides higher resistance to image processing attacks. In this approach, the watermark is embedded in the mid frequency band of the DCT blocks carrying low frequency components and the high frequency sub band components remain unused. Watermark is inserted by adjusting the DCT coefficients of the image and by using key. Watermark can then be extracted using the same private key without resorting to the original image. However, the robustness and value of PSNR were found not satisfactory.

Hong et al. [9] proposed a robust digital watermarking scheme for copyright protection of digital images based on sub-sampling. The watermark is a binary image, which is embedded in discrete transform coefficient of the host image and not used in the original image. In this scheme, chaotic map was used in watermarked image. The result of watermark image is good and robust to attack.

Xia et.al [10] proposed a watermarking scheme based on the Discrete Wavelet Transform (DWT). The watermark, modeled as Gaussian noise, was added to the middle and high frequency bands of the image. The decoding process involved taking the DWT of a potentially marked image. The watermarks were extracted and correlated with sections of the original watermark. If the cross-correlation is more than threshold, then the watermark gets detected. Otherwise, the image was decomposed into finer and finer bands until the entire, extracted watermark was correlated with the entire, original watermark. Performance analysis shows that DWT is more robust to attack than DCT.

Akhil et al. [11] proposed Discrete Wavelet Transform watermarking. The watermark embeds into salient features of the original image using alpha blending technique. The embedding and extraction of watermark is depend only on the value of alpha. All the results obtained for the recovered images and watermark are identical to the original images.

Luo et al. [12] proposed a fast and robust JPEG domain image watermarking method that utilizes spatial masking.
The watermarking algorithm is implemented in the compressed domain, and can also be implemented efficiently for real-time images. In order to achieve a real-time watermarking capability, the proposed method avoids several computation steps associated with JPEG compression. Experiment result shows that the watermarked image is robust to JPEG compression, different kinds of noise and image cropping attacks.

Kundur et al. [13] decomposed binary logo through DWT. The watermark is scaled by a salience factor, computed on a block by block basis, depending on local image noise sensitivity. It is then repeatedly added to the sub-bands of DWT decomposition of host image. Visual masking is thus exploited up to only block resolution. A binary code is embedded by suitably quantizing the coefficients of detail bands. For watermark recovery, the embedded binary code is estimated by analyzing coefficients quantization. Hu et al. [14] proposed an invisible watermarking technique based on DWT in the dual watermarking system to provide additional protection for visibly watermarked images. This paper describes the properties and requirements of such an invisible watermark, and then, gives the details of the watermarking algorithm. The experimental results have shown that the proposed algorithm is very effective to verify the ownership of visibly watermarked image [1519].

Most methods found in literature are highly complex and involve multiple execution stages. This paper presents a simple methodology to hide a grayscale image within another grayscale image using 3-level DWT and blending technique. This is tested against different attacks such as frequency attack (like JPEG compression, Gaussian noise, blurring, and median filtering) and geometrical attack (like rotation).

\section{Discrete WAVELET TRANSFORM (DWT)}

Discrete Wavelet transform (DWT) is a mathematical tool for hierarchically decomposing an image [9]. It is useful for processing of non-stationary signals. The transform is based on small waves, called wavelets, of varying frequency and limited duration. Wavelet transform provides both frequency and spatial description of an image. Unlike conventional Fourier transform, temporal information is retained in this transformation process. DWT is the multi-resolution description of an image the decoding can be processed sequentially from a low resolution to the higher resolution [10]. The DWT splits the signal into high and low frequency components and the high frequency part contains information about the edge components, while the low frequency part is split again into high and low frequency parts. [19-25]. In two dimensional applications, for each level of decomposition, we first perform the DWT in the vertical direction, followed by the DWT in the horizontal direction. After the first level of decomposition, there are 4 sub-bands: LL1, LH1, HL1, and HH1. For each successive level of decomposition, the LL sub-band of 
the previous level is used as the input. To perform second level decomposition, the DWT is applied to LL1 band which decomposes the LL1 band into the four sub-bands LL2, LH2, HL2, and HH2.

To perform third level decomposition, the DWT is applied to LL2 band which decompose this band into the four sub-bands - LL3, LH3, HL3, HH3. This results in 10 sub-bands per component. LH1, HL1, and HH1 contain the highest frequency bands present in the image tile, while LL3 contains the lowest frequency band. The threelevel DWT decomposition is shown in Fig.1.

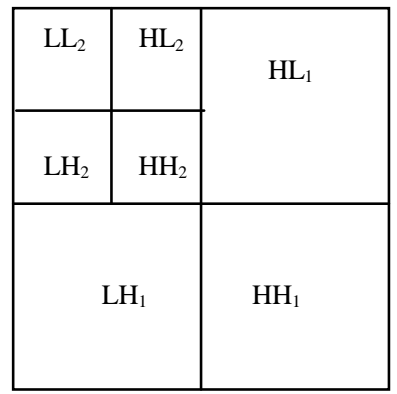

Fig. 1: 3-Level discrete wavelet decomposition.

DWT is currently used in a wide variety of signal processing applications, such as in audio and video compression, removal of noise in audio, and the simulation of wireless antenna distribution [8-12]. The decomposition level is very important factor in digital image watermarking.

\section{WATERMARK ATTACK}

To test the robustness of the proposed algorithm against attacks, watermarked image tested with different attacks like- JPEG compression, Gaussian noise, median filtering, blurring and rotation attack [16]. The JPEG Compression is applied with quality factor where the quality means the amount of degradation in the image to indicate the robustness of the proposed schemes against JPEG compression. The Gaussian noise is applied over the watermarked image with zero mean and 0.0002 variance, where the variance of the noise is a function of the image intensity values in the watermarked image. The Median filter searches the radius of a pixel selection for pixels of similar brightness, discarding pixels that differ too much from adjacent pixels, and replaces the center pixel with the median brightness value of the searched pixels. The blur filter smoothen transitions by averaging the pixels next to the hard edges and shaded areas in an image. Rotation applying a small degree of rotation on the watermarked image will lead to a full damage to the watermark information [22-25].

\section{Proposed Watermarking Technique}

DWT technique is proposed as a new watermarking algorithm. The algorithm is divided into two main parts, watermark embedding and watermark extraction.

\section{A. Watermark Embedding process}

In this stage, the gray scale host image is read and 2D, and 3-level DWT is applied to the image which decomposes image into low frequency and high frequency components. Similarly, 2D and 3-level DWT is also applied to the watermark image which is to be embedded in the host image. The technique used for inserting the watermark is alpha blending $[11,15]$. In this technique the decomposed components of the host image and the watermark are multiplied by a scaling factor and are added. Since the watermark embedded in this paper is perceptible in nature or visible, it is embedded in the low frequency approximation component of the host image. The watermarked image is obtained as:

$$
\mathrm{WMI}=\mathrm{k}^{*}(\mathrm{LL} 2)+\mathrm{q}^{*}(\mathrm{WM} 2)
$$

where $\mathrm{WMI}=$ low frequency component of watermarked image, LL3 = low frequency component of the original image obtained by 3-level DWT, WM3 = low frequency component of Watermark image, and $\mathrm{k}, \mathrm{q}=$ Scaling factors for the original image and watermark respectively [17].

After embedding the cover image with watermark image, 3-level Inverse discrete wavelet transform is applied to the watermarked image coefficient to generate the final secure watermarked image. Fig. 2 shows watermark embedding process

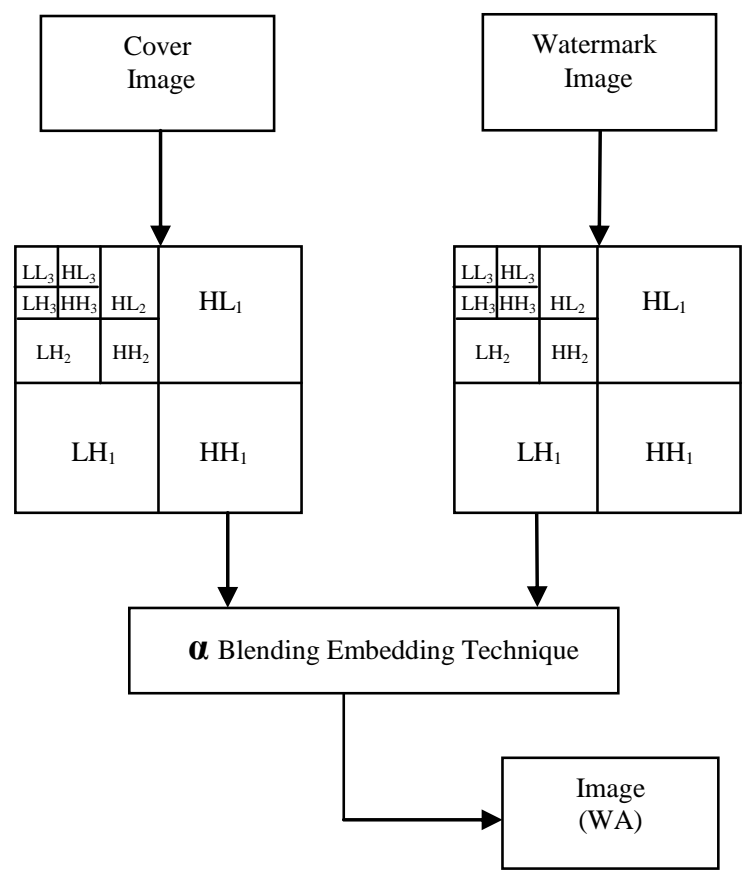

Fig. 2: Watermark Embedding Process.

\section{B. Watermark Extraction process}

Now, 3-level DWT is applied to watermarked image and cover image which decomposes the image into subbands. The watermark is recovered from the watermarked image by using alpha blending: 


$$
\mathrm{RW}=(\mathrm{WMI}-\mathrm{k} * \mathrm{LL} 3)
$$

where RW= Low frequency approximation of recovered watermark, LL3= low frequency approximation of the original image, and $\mathrm{WMI}=$ low frequency approximation of watermarked image.

After extraction process 3-level Inverse discrete wavelet transform is applied to the watermark image coefficient to generate the final watermark extracted image. Fig. 3 shows the final watermark extraction process.

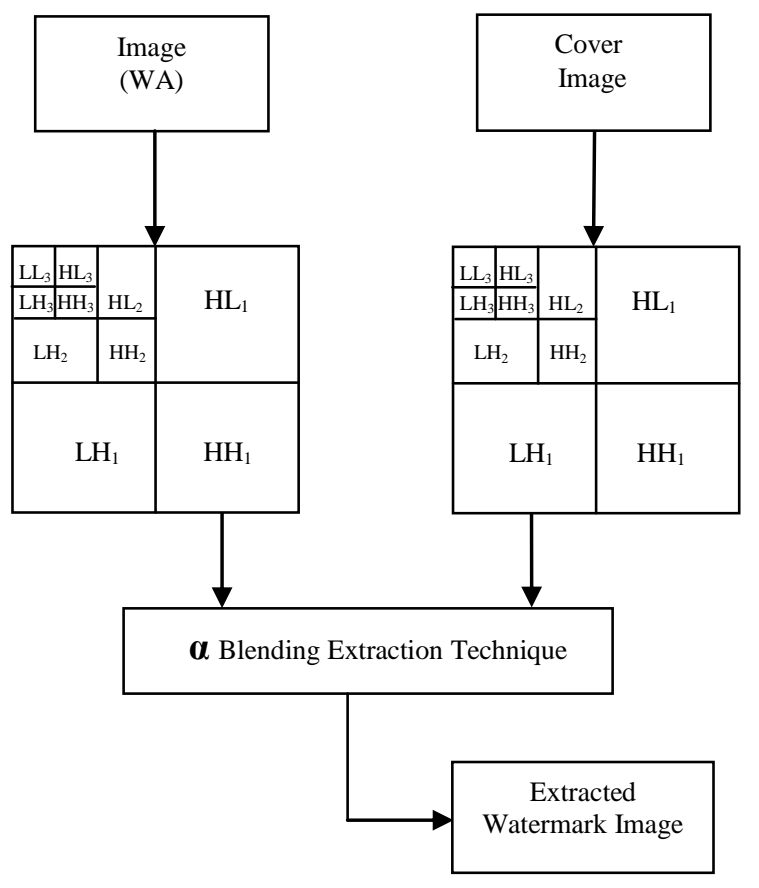

Fig. 3: Watermark extraction technique.

\section{EXPERIMENTAL RESULTS}

We have used grayscale image of Fruits as cover image and the peppers image as the watermark which are shown in Fig. 4 (a) and Fig. 4 (b) respectively. Both the images are of equal size of $256 \times 256$.

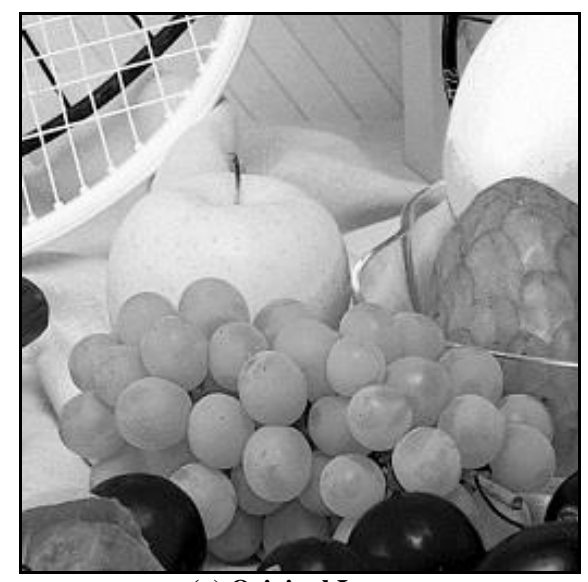

(a) Original Image

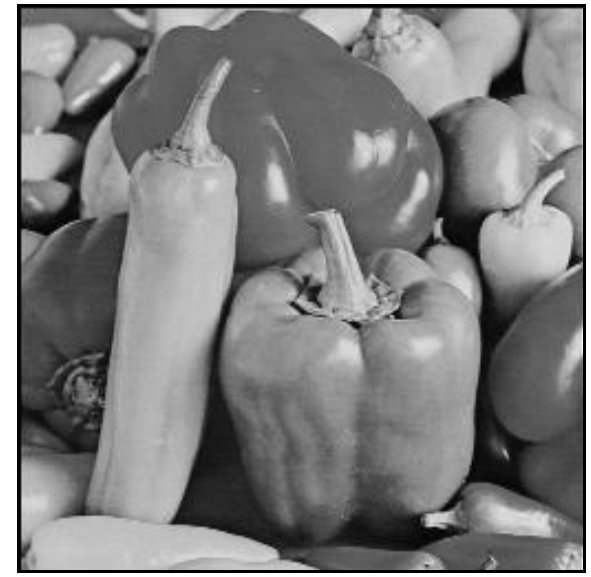

(b) Watermark

Fig . 4: Original and Watermark image

For embedding of watermark in the original image the selected value of scaling factor $\mathrm{k}$ is 0.98 and of $\mathrm{q}$ is 0.009 . Fig. 5 and Fig. 6 depict the watermarked image and recovered image respectively using 3-level DWT.

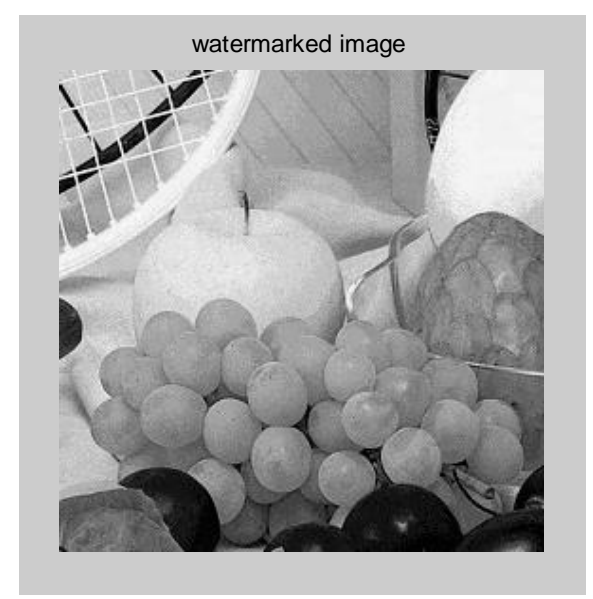

Fig. 5: Watermarked images using 3-level DWT.

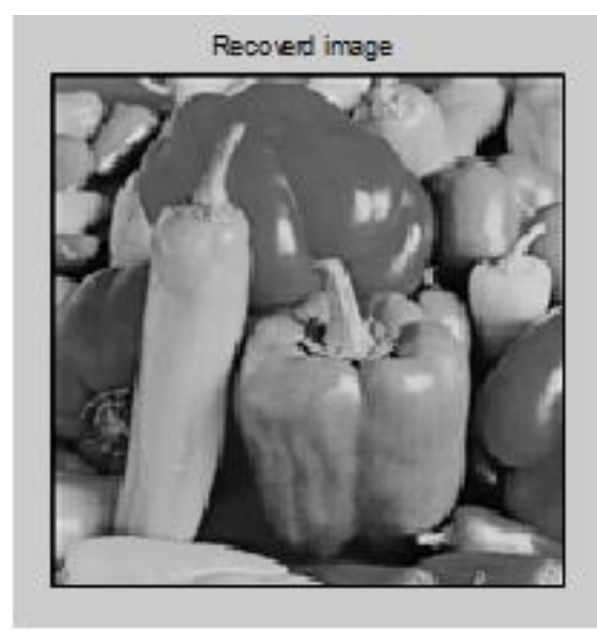

Fig. 6: Recovered image.

We tested the performance of proposed methods by applying different attacks like- JPEG Compression, Gaussian noise, Median filtering, Blur filter and Rotation. 
Table I and Table II highlight the performance of 3-level DWT watermarking under different attacks.

Table 1 Comparison of CC, MSE and PSNR values of watermarked image under different attacks.

\begin{tabular}{|c|c|c|c|}
\hline Types of Attack & CC & MSE & PSNR \\
\hline No attack & 0.9999 & 01.808 & 45.557 \\
\hline $\begin{array}{c}\text { JPEG } \\
\text { Compression }\end{array}$ & 0.9876 & 96.581 & 28.282 \\
\hline $\begin{array}{c}\text { Gaussian noise } \\
\text { Median filtering }\end{array}$ & 0.9225 & $6.10 \mathrm{e}+02$ & 20.272 \\
\hline Blur Effect & 0.995 & $1.16 \mathrm{e}+02$ & 27.497 \\
\hline Rotation & 0.999 & 02.841 & 43.596 \\
\hline
\end{tabular}

Table 2. Omparison of Cc, Mse and Psnr Values of the Recovered Image under Different Attacks

\begin{tabular}{|c|c|c|c|}
\hline Types of Attack & CC & MSE & PSNR \\
\hline No attack & 0.9999 & 05.347 & 45.557 \\
\hline $\begin{array}{c}\text { JPEG } \\
\text { Compression }\end{array}$ & 0.9875 & 96.471 & 28.287 \\
\hline Gaussian noise & 0.9225 & $6.10 \mathrm{e}+02$ & 20.272 \\
\hline Median filtering & 0.9852 & $1.16 \mathrm{e}+02$ & 27.497 \\
\hline Blur Effect & 0.9946 & 47.556 & 31.358 \\
\hline Rotation & 0.9999 & 02.841 & 43.596 \\
\hline
\end{tabular}

It can be observed that 3-level DWT algorithm is robust to different attacks. Under normal conditions, when there is no attack, the verification accuracy has highest performance and least against Gaussian noise. The experiment results of Level-3 DWT watermarking method under JPEG Compression, Gaussian noise, Median filtering, Blur filter and Rotation attack have been compared in terms of PSNR value in Fig. 7.

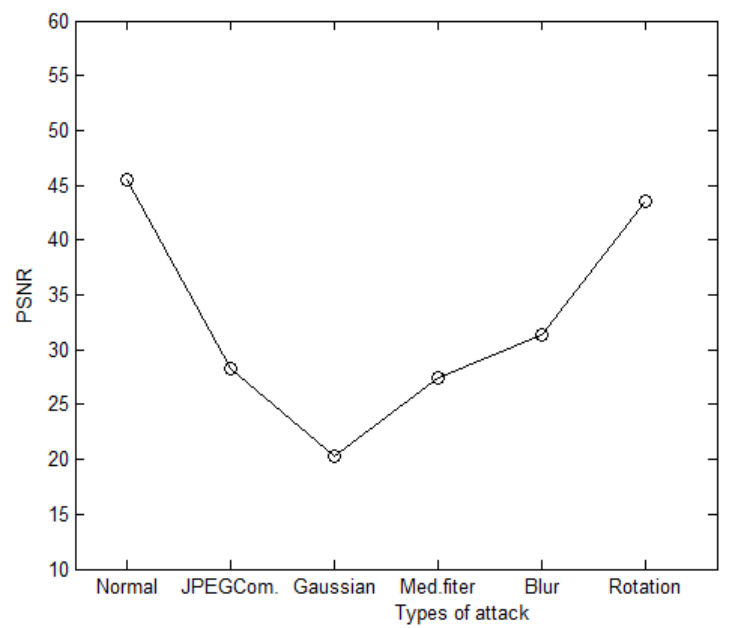

Fig 7. Comparison of PSNR value of watermarked images under different attack

\section{CONCLUSIONS}

This paper presents a complete overview of Digital Image Watermarking techniques in transform domain (3level DWT). This technique can embed the invisible watermark into salient features of the image using alpha blending technique. Experiment result shows that the quality of the watermarked image and the recovered watermark depend only on the scaling factors $\mathrm{k}$ and $\mathrm{q}$. The DWT watermarking is resilient to frequency attacks and least robust to Gaussian noise.

\section{REFERENCES}

[1] Vidyasagar M. Potdar, Song Han, Elizabeth Chang, "A Survey of Digital Image Watermarking Techniques," $3 r d$ International Conference on Industrial Informatics (INDIN), pp. 709-716, 2005.

[2] Ibrahim, R. and Kuan, T. S., "Steganography Imaging (SIS): Hiding Secret Message inside an Image," Proceedings of the World Congress on Engineering and Computer Science, San Francisco, USA, 2010.

[3] Nikita Kashyap and Sinha G.R., "Image watermarking using 2-level DWT", Advances in Computational Research, Vol. 4, Issue 1, pp.42-45, 2012.

[4] L. Robert, T. Shanmugapriya, "A Study on Digital Watermarking Techniques," International Journal of Recent Trends in Engineering, 2009.

[5] G. Rosline Nesa Kumari, B. Vijaya Kumar, L. Sumalatha, and Dr V. V. Krishna, "Secure and Robust Digital Watermarking on Grey Level Images," International Journal of Advanced Science and Technology, 2009.

[6] Darshana Mistry, "Comparison of Digital Watermarking methods," 21st Computer Science Seminar SA1-T1-7, IJCSE, 2010.

[7] Baisa L. Gunjal, R.R. Manthalkar, "An overview of transform domain robust digital image watermarking algorithms," Journal of Emerging Trends in Computing and Information Sciences, 2010.

[8] Blossom Kaur, Amandeep Kaur, Jasdeep Singh, "Steganographic Approach for hiding Image in DCT Domain," International Journal of Advances in Engineering \& Technology, July 2011.

[9] W. Hong and M. Hang, "Robust Digital Watermarking Scheme for Copy Right Protection," IEEE Trans. Signal Process, vo.12, pp. 1- 8, 2006.

[10] X. Xia, C. Boncelet, and G. Arce, "A Multiresolution Watermark for Digital Images," Proc. IEEE Int. Conf. on Image Processing, Oct. 1997.

[11] Akhil Pratap Shing, Agya Mishra, "Wavelet Based Watermarking on Digital Image," Indian Journal of computer Science and Engineering, 2011.

[12] W. Luo, G.L. Heileman "A fast and robust watermarking method for JPEG images," Computer Modelling \& New Technologies, Vol. 8, No.1, pp. 39-47, 2004.

[13] D. Kundur and D. Hatzinakos, "Digital Watermarking using Multiresolution Wavelet Decomposition," Proceedings, IEEE International Conference Acoustic, Speech, Signal Processing, 1998.

[14] Nilanjan Dey, Anamitra Bardhan Roy, Sayantan Dey, “A novel approach of color image hiding using RGB color planes and DWT," International Journal of Computer Applications, 2011.

[15] Baisa L. Gunjal, R.R. Manthalkar, "An Overview of Transform Domain Robust Digital Image Watermarking Algorithms," Journal of Emerging Trends in Computing and Information Sciences, Vol. 2, No. 1, pp. 37-42, 2011. 
[16] R. G. van Schyndel, A. Z. Tirkel, and C. F. Osborne, "A digital watermark," in Proc. IEEE Int. Conf. Image Processing (ICIP), 1994.

[17] Nikita Kashyap and G.R.Sinha, "Image Watermarking Using 3-Level Discrete Wavelet Transform (DWT)", International Journal of Modern Education and Computer Science, Vol. 3, pp. 50-56, 2012.

[18] Kunal D Megha, Nimesh P Vaidya and Ketan Patel, "International Journal of Advanced Research in Computer and Communication Engineering", Vol.2, No.6, pp. 2397 2402, 2013.

[19] V.Santhi and Dr. Arunkumar Thangavelu, "DWT-SVD Combined Full Band Robust Watermarking Technique for Color Images in YUV Color Space", International Journal of Computer Theory and Engineering, Vol.1, No.4, pp.1793-8201, 2009.

[20] Sangeeta Madhesiya and Shakil Ahmed, “Advanced Technique of Digital Watermarking based on SVD-DWTDCT and Arnold Transform", International Journal of Advanced Research in Computer Engineering \& Technology (IJARCET), Vol.2, No 5, pp.1918-1923, May 2013.

[21] Yusuf Perwej , Firoj Parwej and Asif Perwej," Copyright Protection of Digital Images Using Robust Watermarking Based on Joint DLT and DWT" International Journal of Scientific \& Engineering Research Vol.3, No.6, pp.1-9, June-2012.

[22] Ghazali Bin Sulong, Harith Hasan , Ali Selamat3 , Mohammed Ibrahim and Saparudin, "A New Color Image Watermarking Technique Using Hybrid Domain", IJCSI International Journal of Computer Science Issues, Vol. 9, No 1, pp.109-114,November 2012.

[23] Nadia Idrissi, Ahmed Roukh, Lhoussaine Masmoudi, Mohamed Radouane And Rochdi Messoussi, “A Robust Digital Watermarking Technique using DWT-DCT and Statics blocks" IJCSI International Journal of Computer Science Issues, Vol. 10, No 1, pp.45-48,March 2013.

[24] Mahasweta J.Joshi, Prof. Zankhana H.Shah and Keyur N.Brahmbhatt, "Watermarking in DCT-DWT Domain", (IJCSIT) International Journal of Computer Science and Information Technologies, Vol.2, No.2, pp. 717-720, 2011.

[25] Sarvesh Kumar Yadav, Shital Gupta and Vineet richariya, "Digital Image Watermarking Using DWT and SLR Technique Against Geometric Attacks", International Journal of Computer Technology and Electronics Engineering (IJCTEE) Vol.2, No.1, pp.43-47, 2012.

\section{Authors Profile}

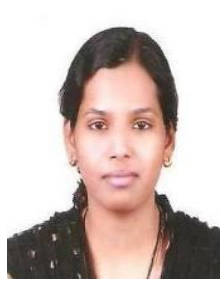

Asma Ahmad has received the BE degree from Chouksey Engineering College Bilaspur in Electronics and Telecommunication Engineering in 2008. She is working towards the M.E. degree in Communication from Shri Shankaracharya Technical Campus Bhilai. Her areas of interests include digital image processing, embedding system and biometric system.

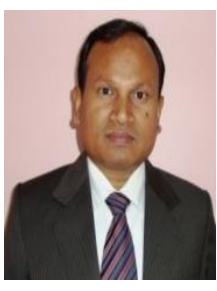

G.R.Sinha is Professor of Electronics \& Telecomm. and Associate Director in Faculty of Engineering and Technology of Shri Shankaracharya Group of Institutions, Shri Shankaracharya Technical Campus Bhilai of Chhattisgarh Swami Vivekanand Technical University India. He obtained his B.E. (Electronics) and M.Tech. (Computer Technology) from National Institute of Technology, Raipur. He received Gold Medal for obtaining first position in the University. He received his Ph.D. in Electronics \& Telecommunication from Chhattisgarh Swami Vivekanand Technical University, Bhilai. He is Dean of Faculty and Executive Council Member Chhattisgarh Swami Vivekanand Technical University, Bhilai.

He has published 134 research papers in various international and national journals and conferences. He is active reviewer and editorial member of more than 12 international journals such IEEE Transactions on Image Processing, as Applied Physics Research of Canada, IBSU Scientific Journal of Georgia, Scientific Journals International (SJI) of USA, Computer Science Journals of Malaysia etc. He has reviewed research papers of many International Conferences including IEEE conferences. He is recipient of many awards like Emerging Chhattisgarh Award 2013: Best Academician, Engineer of the Year Award 2011, Young Engineer Award 2008, Young Scientist Award 2005, IEI Expert Engineer Award 2007, nominated for ISCA Young Scientist Award 2006 and awarded Deshbandhu Merit Scholarship for 05 years. He has been selected as Distinguished IEEE Lecturer in IEEE India council for Bombay section. He has been elevated to Senior Member of IEEE.

He has delivered many keynote speeches and chaired many technical sessions in international conferences in Singapore, Mumbai and Nagpur \& across the country. He is member of signal processing society of IEEE, IACSIT \& SIE Singapore and also of many national professional bodies like IETE, ISTE, CSI, ISCA, and IEI. He is member of various committees of the University and has been Vice President of Computer Society of India for Bhilai Chapter for two consecutive years. He has authored Five Books including recently published on Biometrics: Concepts and Applications published by Wiley India, a subsidiary of John Wiley, ISBN: 978-81-265-3865-2. His research interest includes Digital Image Processing and it applications in biometric security, forensic science, pattern recognition, early detection of breast cancer, content retrieval of underwater imaging, Vehicle license plate recognition etc.

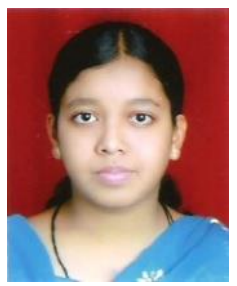

Nikita Kashyap received her B.E. in Electronic \& Telecommunication Engineering from Institute of Technology, Guru Ghasidas Central University, Bilaspur in 2010 and M.E. in Communication from Shri Shankaracharya Group of Institutions, Bhilai in 2012. She has published 3 research papers in journals. She is working as Assistant Professor in Lakhmi Chand Institute of Technology, Bilaspur, India. Her areas of interests are image processing, data hiding and image watermarking.

How to cite this paper: Asma Ahmad, G.R.Sinha, Nikita Kashyap,"3-Level DWT Image Watermarking Against Frequency and Geometrical Attacks", IJCNIS, vol.6, no.12, pp.58-63, 2014. DOI: 10.5815/ijcnis.2014.12.07 